\title{
Bridging the Problem-Based Learning Gap for the \\ Pre-Medical Student: Lessons Learned from Physicists Maxwell and Einstein
}

Diana R. Cundell* and Edward Santilli 


\section{- Abstract}

Our pre-medical curricula have changed little since Flexner identified eight core classes in 1910. Discussion has continued to refine what science mastery is needed to become a medical school matriculant, but there remains a hierarchy of courses designed to produce an effective score on the Medical College Admissions Test. In many countries, students go from siloed learning as undergraduates to integrated, problem-based learning $(\mathrm{PBL})$ as graduate students. Unfortunately, without prior exposure to the PBL model, first-year medical students struggle and may fail to continue. In physics, the great minds of Maxwell and Einstein unified existing ideas, creating new, simpler theories. The idea of unification lies at the forefront of physics and, following suit, the future physician needs to deconstruct the boxed courses to see the body as a single entity. We would argue that upper-level pre-medical students should prepare to perform well in an interdisciplinary course such as pathology, which is typically part of the first-year medical school curriculum. Such "early exposure" would increase the students' comfort and engagement with their future studies and fortify active, analytical learning.

\section{- Introduction}

In the seventh century, Isidore of Seville recommended that a physician should receive a "broad pre-medical education with literature, rhetoric, dialectic, arithmetic, geometry, music and astronomy." By 1910, Abraham

Flexner had expanded this to a two-year curriculum of individual anatomy, pathology, pharmacology, ${ }^{2}$ and botany ${ }^{3}$ courses. Evaluation of knowledge, logic and understanding began in 1928 with the earliest form of the Medical College Admissions Test (MCAT) ${ }^{2}$, and pre-medical curricula have been bound to this ever since. ${ }^{2,4}$ In 2015, the MCAT switched to analytical skills questions and added a section on psychological and social sciences, ${ }^{2}$ which benefitted students matriculating from liberal arts institutions, such as our own. ${ }^{4}$

The pre-medical curriculum was originally designed to "free up" the medical school preclinical components ${ }^{1}$ and match the MCAT. ${ }^{2}$ Keeping

Division of Health Professions, College of Science Health and the Liberal Arts, Jefferson (Philadelphia University and Jefferson University), Philadelphia, Pennsylvania.

*Address for correspondence: Prof. Diana R. Cundell, Pre-Medical Studies Program Director and Clinical Coordinator, Division of Health Professions, College of Science Health and the Liberal Arts, Jefferson (Philadelphia University and Jefferson University), 4201 Henry Avenue, Philadelphia, Pennsylvania 19144. E-mail: cundelld@philau.edu

๑) Diana R. Cundell and Edward Santilli 2018; Published by Mary Ann Liebert, Inc. This article is available under the Creative Commons License CC-BY-NC (http://creativecommons.org/licenses/by-nc/4.0). This license permits non-commercial use, distribution, and reproduction in any medium, provided the original work is properly cited. Permission only needs to be obtained for commercial use and can be done via RightsLink. 
up with the new MCAT scope means pre-medical students now need biochemistry and several courses in psychology and sociology, ${ }^{2}$ decreasing their free elective options. ${ }^{5}$ Students know that even free electives can harm their grade point average, ${ }^{1}$ and this often narrows their knowledge diversity still further. Additionally, MCAT mastery requires we "teach for the test," but how much do students really remember? Unlike liberal arts courses, which have a philosophy of integrating course information, ${ }^{4}$ the silos of science curricula mean students make few connections between classes and retain less information as a result. ${ }^{6}$ To address this, some institutions are blending introductory biology with genetics or molecular biology. ${ }^{5}$ But unless the knowledge involves deep learning, it is usually lost within two years of course completion. ${ }^{7}$ Deep learning is created by active strategies involving concept building, repeated exposure to information, rearrangement of information, and connection to other classes or concepts. ${ }^{7}$ Visual memory also provides more cues than any other, and perceptive memory results in effective abstract reasoning. ${ }^{8}$

Making subjects meaningful requires relevance. .,2,4,5,9,10 $^{1,4}$ Some suggest this could be done through the introduction

66 Medical schools have overcome concept building issues by teaching through problem-based learning. 99 of medical genomic and informatics, since these are the basis of future medical treatments. ${ }^{5}$ Others observe that simulated patient experiences, using artificial patients, ${ }^{1,9}$ role playing, ${ }^{4,10,11}$ or exposure to the clinical setting as a volunteer ${ }^{2}$ improve students' interest and knowledge retention. These latter experiences develop the learner's visual memory, ${ }^{8}$ since they now associate a disease condition with a patient rather than a litany of facts.

Medical schools have overcome concept building issues by teaching through problem-based learning (PBL). ${ }^{11,12}$ This "block learning" approach involves small group problem solving, learning together, and then presenting to a larger audience. ${ }^{11,12}$ Begun in 1969, PBL connects science to clinical studies and makes subject matter more meaningful, ${ }^{11}$ especially to female students, ${ }^{13}$ plus it improves exam performance. ${ }^{13} \mathrm{PBL}$ represents an advanced form of the active learning that has always driven knowledge retention and exam performance for STEM majors. ${ }^{4,14}$ A good grounding for $\mathrm{PBL}$ is also provided by undergraduate research and laboratory experiences requiring synthesis and analysis of data. ${ }^{15}$ Although usually effective, PBL has some flaws, as it favors those with previous topic 
knowledge and fails to identify weak students whose contributions may be less than stellar. ${ }^{12}$ Also, first-year students often struggle with this type of transdisciplinary, non-classroom regimented type of learning and may underperform or fail as a result ${ }^{16}$ probably due to unfamiliarity with this format.

In non-U.S. medical schools, medical degrees last four to six years, with the first half being preclinical studies, such as our undergraduate pre-medical degree, and the second half being clinical studies. ${ }^{13,17}$ Students are immersed in PBL throughout this time and achieve mastery after three years of training. ${ }^{16} \mathrm{PBL}$ has been successfully used to integrate theories in individual undergraduate courses such as biochemistry ${ }^{18}$ and physics, ${ }^{19}$ so why not in an interdisciplinary course? Would taking such an upper-level interdisciplinary PBL course as an undergraduate provide a similar "knowledge bridge" to medical school philosophy as does taking high-school AP credits? ${ }^{20}$ If the answer is yes, then the other issue is whether junior and senior pre-medical students would be able to master a PBL interdisciplinary biology, biochemistry, and clinical course successfully.

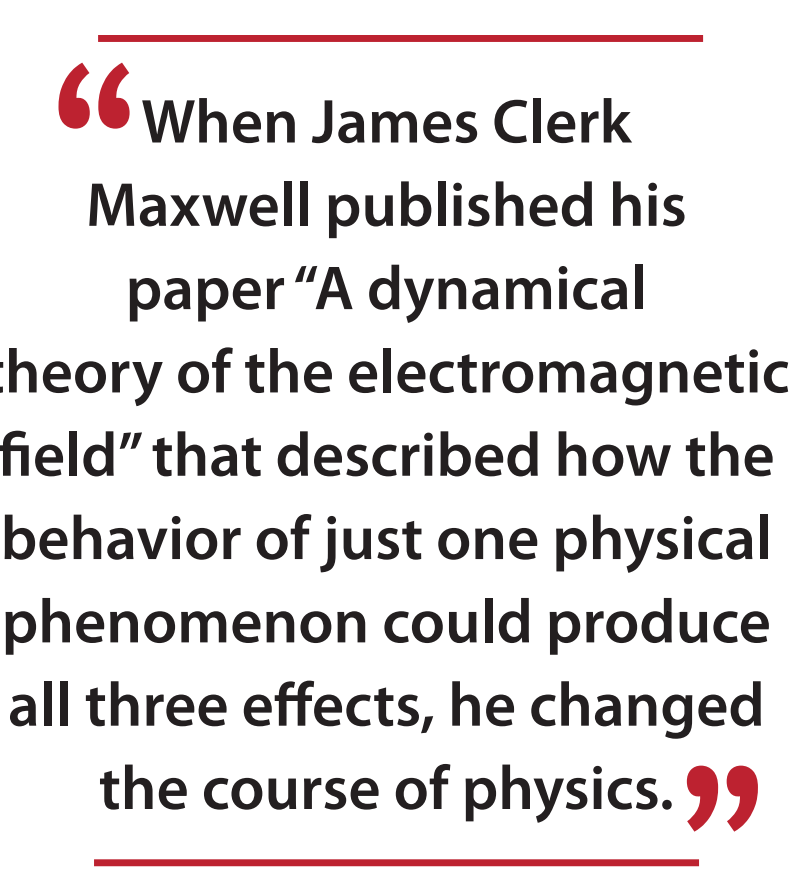

In tackling this thorny problem, our attention naturally turned to how past science luminaries have unified disparate concepts. Prime examples came not from biology but from the physicists James Clark Maxwell and Albert Einstein who unified physical phenomena to explain and expand our understanding of electromagnetism and gravity, respectively. In this article, we will examine how lessons learned from Maxwell and Einstein assisted us in understanding the creation of interdisciplinary theory, as well as presenting a case example for biology: pathology for the undergraduate.

\section{Lessons Learned from Maxwell and Einstein}

Prior to the 1860s, the electric field, magnetic field, and light were considered three distinct physical phenomena, each with their own set 
of rules that govern their behavior. These rules were numerous, small in scope, and often contradictory. When James Clerk Maxwell published his paper "A dynamical theory of the electromagnetic field"21 that described how the behavior of just one physical phenomenon could produce all three effects, he changed the course of physics. Maxwell integrated all the available information and deduced that one set of non-contradictory rules governed the electric field, magnetism, and light. This allowed scientists to manipulate the newly dubbed electromagnetic field to exhibit new effects and to utilize light in totally novel ways.

By converting numerous complex laws into a few concise laws, he discovered sensible explanations for the phenomena at hand, greatly benefiting students. As was said about Maxwell's treatise on the electromagnetic field, "the full meaning of Ohm's law becomes clear; electromotive force, difference of potential, resistance, current, capacity, lines of force, magnetization and chemical affinity were measurable, and could be reasoned about, and calculations could be made about them with as much certainty as calculations in dynamics." ${ }^{22}$ Alongside this benefit, scientists can probe deeper into the details of the theory. They can ask new questions and uncover new ways to utilize light and electromagnetism. No one at the time was more conscious of this than Albert

Einstein. He looked up to the work of Maxwell ${ }^{23}$
66 Prior to the 1900s, there were many complex theories about how light traveled, but to achieve consistency, there needed to be a single medium that all objects, including light, traveled through. 99 for precisely this reason, and it encouraged him to maintain a holistic view of the current state of physics.

Prior to the 1900s, there were many complex theories about how light traveled, but to achieve consistency, there needed to be a single medium that all objects, including light, traveled through. Although there was no evidence of this medium's existence, it was dubbed the "luminiferous aether," 24 and its sole purpose was to help make sense of the otherwise inexplicable experimental results. However, the aether itself was difficult to explain because its presence demanded a certain experimental evidence that flew in the face of the experimental results of the time. ${ }^{25,26}$ This led a small number of scientists, including Einstein, to reject the aether, despite its widespread acceptance. 
One of the experiments that assumed the notion of aether was led by Michelson and Morley who set out to compute how fast the Earth traveled through this medium by calculating the speed of light when cast in different directions. ${ }^{27}$ They theorized that if the Earth is traveling around the Sun at approximately $100,000 \mathrm{~km} / \mathrm{h}$, then there would be a noticeable "wind" caused by the Earth's motion through the aether. This could be detected by measuring the speed of light when shined into or against this aether wind. However, Michelson and Morley observed no difference in the speed of light as they cast it in different directions. Instead of rejecting the aether at this point, many scientists altered the aether theory in to make it more complicated and still fit the observations. ${ }^{28,29}$

Einstein, on the other hand, applied a series of logical challenges that he called gedanken (German for "thoughts") to the experimental results and realized that there was only one realistic conclusion: time and space were connected in a non-trivial way so that light always travels the same speed. ${ }^{30}$ In true Maxwellian fashion, Einstein noted that the two entities of space and time were realizations of one phenomenon, aptly dubbed spacetime. Time is dependent on how we move through space, and gravity is nothing more than an interesting manifestation of spacetime. Furthermore, Einstein provided several tests of his theories and each provided further validation of what was to become the General Theory of Relativity. ${ }^{31}$

66 Applying this to biology, studies unifying components of science and clinical studies needed to identify and diagnose disease would be advantageous in producing a seamless approach to medicine. 99

So, what can we learn from Maxwell's and Einstein's strategy? They both used logical deductions to identify common ground, creating a conceptual thread to unify several ideas. By producing this "thread," they allowed the connections to be established into a whole, which became more than the sum of its parts. In turn, by understanding these unified physics concepts, the parts have greater significance to those studying them, that is, they provide deep learning. Applying this to biology, studies unifying components of science and clinical studies needed to identify and diagnose disease would be advantageous in producing a seamless approach to medicine. The next part of this article will discuss the success we have had with a pathology course that also provides basic empathic and professional skills. ${ }^{4}$ 


\section{Pathology: An Undergraduate Case Study in Interdisciplinary Biology}

Originally established in 1997 at our Institution, the undergraduate pathology course was developed with the assistance of a former pre-medical alumnus, Dr. Brandon Francis, to develop students' skills to a level just at or below that of a first-year medical student upon the completion of the course. Since its inception, several features of the pathology course have supported the development of early PBL in students who have at least already taken anatomy, microbiology, and biochemistry. First, lecture information is integrated, so there is less sense of being in specific organ systems or that the series of evaluation sessions (exams) mean that forgetting previous information will still allow success in the course. This breaks down the anatomy silos to perceive the human body as an integrated system that is more than the sum of its parts. Also, even intelligent, curious students are often amazed to discover that when disease affects the heart, for example, there is a consequent deleterious effect on the lungs and vice versa! This is an important lesson to an educator, as associations are not always obvious. Second, students work in teams of three or four to complete a series of eight "mini PBL" during which they research a "case" complete with blood work, pathology reports, X-rays, magnetic resonance imaging, and so on. These are then presented to the class for further discussion and expansion and to allow students to develop a realistic perspective of differential diagnosis. Students are not provided any URL or other links to analyze this information, which requires them to hone their information literacy skills. In addition, they are asked to develop their diagnoses as they proceed through the case, that is, change their minds as new results are provided. Justification for evolving decisions mirrors the real-world situation and develops deep analytical abilities. Third, the exam material involves little to no direct factual questioning. Instead, a series of mini cases (resembling the PBL above) are presented, which require students to make judgments of a situation, discuss issues, identify needed information and explain their reasoning behind treatment strategies. Examinations provide an assessment of skill development and increase in difficulty throughout the semester. By the third and final examinations, 
students are being asked to think at a level just below that of a first-year medical school student. Fourth, direct connections between lecture material and "real-world" clinical presentations are supported through role play, in which all students act as clinician and patient to simulate disease conditions. Described elsewhere, ${ }^{4}$ this develops empathic skills and provides an added benefit to the experience. Students in the class will already have completed between 100 and 200 clinical shadowing hours, depending on whether they are pre-medical or physician assistant (PA) majors, and this activity allows them to "dig deep" into what they have observed and learned from external healthcare agencies.

Although students have various aptitudes for this course, pathology allows students to assemble the science jigsaw to see the "big picture," and this can have several long-term benefits. In annual questionnaires, $>75 \%$ have consistently indicated a substantial increase in their analytical and synthetic abilities, that is, they can take knowledge and apply it

66 Students in our PA program who take the course have been shown to receive significantly higher grades in their master's level pathophysiology course. 99 using more than a one-step process (unpublished data). Such skills create true subject inquiry and develop students who begin to think outside the box. Students who have gone on to medical school have described pathology as "the most influential course in developing my PBL abilities" stating that "my fellow students feel I am at an advantage in the class because of this course and my ability to look at many aspects of disease and illness simultaneously." Finally, students in our PA program who take the course have been shown to receive significantly higher grades in their master's level pathophysiology course, which employs a similar holistic approach to the human body (personal communication from PA Faculty Member Jennifer Anderson).

\section{Conclusions and Further Directions}

Clearly, the MCAT barrier with a new challenge of PBL learning are here to stay in our medical schools, but as teaching faculty and program directors of pre-medical curricula, it is our responsibility to provide methods to help students overcome these hurdles. Changing the individual components of our curriculum to provide a broad-based education has become an increasingly essential value-added proposition - but this discussion is clearly the fuel for a different type of article. Integrating them in a way that 
allows students to see the segue between all they have learned and the clinical world beyond is paramount to ensure they do indeed succeed in an integrated learning system.

Maxwell and Einstein were uniquely brilliant physicists whose minds shaped the understanding of the laws governing the physical sciences into unified theories and concepts. This required a deep understanding of all the fields they were assimilating and involved thought processes that would not be easily replicated during our history. In a smaller way, the creation of an interdisciplinary course faces a primary obstacle of the instructor's knowledge base. In our case, pathology is taught by the single individual responsible for its design, but it could be imagined that in other institutions a team-taught situation might be needed. Depending on the number of faculty needed, content distribution between the science courses involved might be a stumbling block for some institutions, so pathology is not expected to be a "one size fits all."

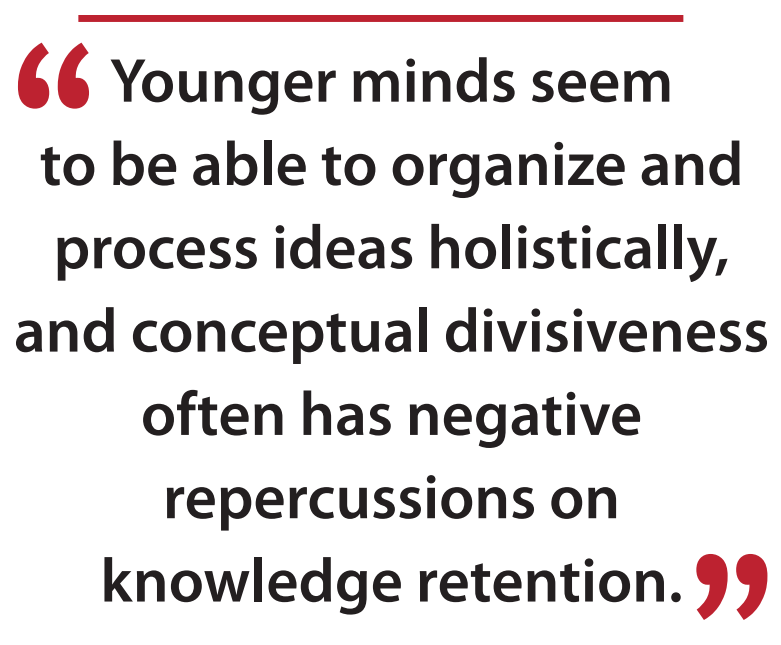

$6 6 \longdiv { \text { Younger minds seem } }$ to be able to organize and process ideas holistically, often has negative repercussions on knowledge retention. 99

Clearly, pathology can be an undergraduate course and provide students with the necessary tools to attack the PBL of graduate school, but should there be others like it? If so, what might they be? Given that much of the information required of the future health professional is likely to be online, it may be that moving away from focus on memorization of minutiae and understanding instead "how to" and "how things fit," that is, providing tools that encourage dynamic thinking may be more appropriate. ${ }^{32}$ Younger minds seem to be able to organize and process ideas holistically, and conceptual divisiveness often has negative repercussions on knowledge retention. ${ }^{11}$ Systems biology, which is being adopted by many undergraduate institutions, would provide this type of unified approach especially for the entering high-school student, ${ }^{32}$ but again the devil is in the detail, and MCAT is still a fact-driven examination. Whether pathology remains an isolated forum or has other courses like it in the pre-medical curriculum remains to be seen, but currently it does fulfill the definition of an interdisciplinary course in that there is a "shift of emphasis to themes as a focus for learning" and that it may be associated with a "loss of reference to individual disciplines or subjects." ${ }^{11}$ In the Harden methodology of curricular integration, this represents the second rung 
from the top of the ladder, and is a point at which the student is taking significantly more responsibility for their learning than the isolated courses at the beginning. ${ }^{11}$

Pre-medical curricula need to evolve to include multi- or interdisciplinary courses to bridge the medical school gap. Adding courses that are analogous to graduate preclinical studies can be a successful strategy. As educators and program directors, it is essential to remember that "A sick patient does not represent a biochemistry problem, an anatomy problem, or a genetics problem or an immunology problem; rather each person is the product of myriad molecular, cellular, genetic, environmental, and social influences that interact in complex ways to determine health and

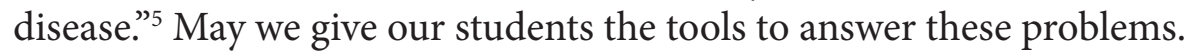

\section{- Acknowledgments}

The authors would like to thank Dr. Brandon Francis (Class of 2001) for his valuable assistance in constructing the pathology course.

\section{- Author Disclosure Statement}

No competing financial interests exist.

\section{References}

1. Kanter SL. Toward a sound philosophy of premed education. Acad Med. 2008. 83 (5): 423-424.

2. Mitchell K, Satterfield J, Lewis RS, et al. The new medical college admissions test: implications for teaching psychology. Am Psychol. 2016. 71 (2): 125-135.

3. Muller D. Reforming premedical education-out with the old, in with the new. New Engl J Med. 2013. 368: 1567-1569.

4. Cundell DR. Culturing the empathic professional. Healthc Transform. 2017. 2 (2): 71-83.

5. Dienstag JL. Relevance and rigor in premedical education. New Engl J Med. 2008. 359 (3): 221-224.

6. Wilkerson L, Stevens CM, Krasne S. No content without context: integrating basic, clinical, and social sciences in a pre-clinical clerkship. Med Teach. 2009. 31: 812-821. 
7. Bacon DR, Stewart KA. How fast do students forget what they learned in consumer behavior? A longitudinal study. J Market Educ. 2006. 28 (3): 181-192.

8. Brady TF, Konkle T, Alvarez GA, et al. Visual long-term memory has a massive storage capacity for object details. Proc Natl Acad Sci U S A. 2008. 105 (38): 14325-14329.

9. Gordon JD, Hayden EM, Ahmed RA, et al. Early bedside care during preclinical education: can technology-enhanced patient simulation advance the Flexnerian idea? Acad Med. 2010. 85: 370-377.

10. Jones F, Passos-Neto CE, Braghroli JFM. Simulation in medical education: brief history and methodology. Princ Pract Clin Res. 2015. 1 (2): 56-63.

11. Atwa HS, Gouda EM. Curriculum integration in medical education: a theoretical review. Intel Prop Rights. 2014. 2 (2): 113.

12. Chang BJ. Problem-based learning in medical school: a student's perspective. Ann Med Surg. 2016. 12: 88-89.

13. Niwa M, Saiki T, Fujsaiki K, et al. The effects of problem-based learning on the academic achievements of medical students in one Japanese medical school, over a twenty year period. Health Prof Educ. 2016. 2: 3-9.

14. Freeman S, Eddy SL, McDonough M, et al. Active learning increases student performance in science, engineering and mathematics. Proc Natl Acad Sci U S A. 2014. 111 (23): 8410-8415.

15. AlAmoudi A. Problem-based learning sessions and undergraduate research: a medical student's perspective and experience. Perspect Med Educ. 2014.3 (1): 56-60.

16. Glew RH. The problem with problem-based medical education: promises not kept. Biochem Mol Biol Educ. 2003. 31 (1): 52-56.

17. Musal B, Gursel V, Taskiran C, et al. Perceptions of first and third year medical students on self-study and reporting processes of problembased learning. BMC Med Educ 2004. 4: 16.

18. Tahan L. The views of undergraduates about problem-based learning applications in a biochemistry course. J Biol Educ. 2015. 49 (2): 116-126.

19. Aziz MS, Nurulazam A, Samsudin MAB, et al. The impact of PBL on undergraduate physics students' understanding of thermodynamics. Int J Acad Res Econ Manag Sci. 2014.3 (4): 100-112.

20. Warne RT. Research on the academic benefits of the advanced placement program: taking stock and looking forward. SAGE Open. 2017. 7 (1). 
21. Maxwell JC. VIII. A dynamical theory of the electromagnetic field. Phil Trans R Soc Lond. 1865. 155: 459-512.

22. Maver W J. Electricity, its history and progress (Vol. X). New York: Encyclopedia Americana Corp., 1918.

23. Einstein A, Hawking SW. A stubbornly persistent illusion: the essential scientific works of Albert Einstein. Philadelphia, PA: Running Press, 2007.

24. Michelson AA, Morley EW. On the relative motion of the Earth and the luminiferous ether. Am J Sci. 1887. 34: 333-345.

25. Miller D. The ether-drift experiments at Mount Wilson solar observatory. Phys Rev. 1922. 19: 407-408.

26. Silberstein L. The relativity theory and ether drift. Science. 1925. 62 (1596): viii

27. Swenson L. The ethereal aether: a history of the Michelson-MorleyMiller aether-drift experiments. Austin, TX: University of Texas Press, 1972.

28. Whittaker ET. A history of the theories of aether and electricity (1st edn.). Dublin, Ireland: Longman, Green and Co., 1910.

29. Janssen M, Stachel J. The optics and electrodynamics of moving bodies. Berlin, Germany: Max-Planck-Institute for the History of Science, 2004.

30. Einstein A. On the electrodynamics of moving bodies. Annalen der Physik. 1905. 17.

31. Einstein A. 1879-1955. The collected papers of Albert Einstein. Princeton, NJ: Princeton University Press, 1987.

32. Hillmer RA. Systems biology for biologists. PLoS Pathog. 2015. 11 (5): e1004786. 